\title{
Oncologic outcomes after immediate breast reconstruction following mastectomy: comparison of implant and flap using propensity score matching
}

Jeong Hyun Ha ${ }^{1 \dagger}$, Ki Yong Hong ${ }^{2 \dagger}$, Han-Byoel Lee ${ }^{3}$, Hyeong-Gon Moon ${ }^{4}$, Wonshik Han ${ }^{3,4}$, Dong-Young Noh ${ }^{3,4}$, Joonho Lim', Sehoon Yoon ${ }^{1}$, Hak Chang ${ }^{1,5}$ and Ung Sik Jin ${ }^{1,5^{*}}$

\begin{abstract}
Background: Although immediate breast reconstruction has been reported to be oncologically safe, no affirmative study comparing the two reconstruction methods exists. We investigated breast cancer recurrence rates in two breast reconstruction types; implant reconstruction and autologous flap reconstruction.

Methods: A retrospective cohort study was performed on propensity score-matched (for age, stage, estrogen receptor status) patients who underwent IBR after mastectomy at Seoul National University Hospital between 2010 and 2014. The main outcomes determined were locoregional recurrence-free interval (LRRFI) and disease-free interval (DFI).

Results: We analyzed 496 patients among 731 patients following propensity score matching (Median age 43, 247 implant reconstruction and 249 flap reconstruction). During median follow-up of 58.2 months, DFI was not different between the two groups at each tumor stage. However, flap reconstruction showed inferior DFI compared to implant reconstruction in patients with high histologic grade $(p=0.012)$, and with high Ki-67 $(p=0.028)$. Flap reconstruction was related to short DFI in multivariate analysis in aggressive tumor subsets. Short DFI after flap reconstruction in aggressive tumor cell phenotype was most evident in hormone positive/Her-2 negative cancer $(p=0.008)$. LRRFI, on the other hand, did not show difference according to reconstruction method regardless of tumor cell aggressiveness.

Conclusion: Although there is no difference in cancer recurrence according to reconstruction method in general, flap-based reconstruction showed higher systemic recurrence associated with histologically aggressive tumors.
\end{abstract}

Keywords: Breast neoplasm, Breast reconstruction, Flap, Implant, Oncologic safety

\section{Background}

A combination of surgical excision, systemic chemotherapy, and radiation therapy are applied in breast cancer to improve the oncologic outcome. Immediate breast reconstruction (IBR) has become the mainstay method of aesthetic and functional improvement following mastectomy for breast cancer [1]. It is essential that breast

\footnotetext{
* Correspondence: usj1011@snu.ac.kr

† Jeong Hyun $\mathrm{Ha}$ and Ki Yong Hong contributed equally to this work.

${ }^{1}$ Department of Plastic and Reconstructive Surgery, Seoul National University Hospital, Seoul, South Korea

${ }^{5}$ Department of Plastic and Reconstructive Surgery, Seoul National University College of Medicine, Seoul, South Korea

Full list of author information is available at the end of the article
}

reconstruction be safe from an oncologic point of view. In other words, IBR should not increase the risk of relapse or hinder subsequent anti-cancer treatment such as adjuvant chemotherapy by causing complications [2]. Previous studies have tried to establish the oncologic safety of IBR, showing that IBR did not increase recurrence rates or delay the detection of recurrence $[3,4]$. However, they did not differentiate the outcomes according to specific IBR methods or tumor histologies $[3,5,6]$. To our knowledge, no one has yet performed a matched case-control study comparing locoregional and distant metastasis rates between reconstruction methods. 
It is well established that breast reconstruction provides psychological benefits and improvements in quality of life [7]. Nevertheless, IBR has traditionally not been recommended over delayed reconstruction [8]. The reluctance to use IBR originates from concerns that IBR might increase the risk of locoregional recurrence and that recurrence might be more difficult to detect after IBR [6]. However, recent reports suggest that IBR is oncologically safe in invasive breast cancer and is practiced widely [3, 4, 9-11]. Although the locoregional recurrence rates after IBR vary across studies, it is commonly accepted that IBR does not significantly increase recurrence. For example, an anecdotal study by Eriksen et al. [6] reported that implant-based IBR did not affect oncologic outcomes including locoregional and distant recurrence. The authors compared locoregional and distant recurrence between patients that underwent implant-based IBR and a mastectomy-only group in this study. Likewise, Howard et al. [3] analyzed oncologic outcomes between patients that underwent TRAM flap-based IBR and a mastectomy-only control group. In the study, there was no difference in the local recurrence rate between the two groups.

Implant-based breast reconstruction is a safe method with favorable outcomes, minimal morbidity, and short operative times. On the other hand, flap-based breast reconstruction is performed using microvascular free flap transfer or pedicled flap transfer. Reports suggest that flap reconstruction has certain advantages over implant-based reconstruction, such as a lower complication rate during adjuvant radiotherapy and better long-term aesthetic outcomes [12-15]. Some surgeons prefer flap reconstruction over implant-based reconstruction when the cancer is in a locally advanced stage, because the former is less likely than the latter to cause complications during adjuvant therapy [16]. In general, patient-specific factors such as breast size, degree of ptosis, comorbidity, age, and patient preference are used to determine the best method of reconstruction for each patient [17].

Although recent reports suggest that IBR does not affect cancer recurrence or detection $[3,5,18]$ there are still oncologic concerns about the use of flap reconstruction [19]. There are no affirmative data comparing locoregional recurrence rates between implant-based reconstruction and flap reconstruction. Although neither method is reported to increase the relapse risk in general, the oncologic safety of flap reconstruction still needs to be more precisely analyzed from diverse perspectives. The aim of this study was to investigate whether there is a difference in locoregional and overall recurrence between those two reconstruction methods in patients with breast cancer who undergo IBR.

\section{Methods}

\section{Patients}

We identified all patients who underwent IBR at Seoul National University Hospital (SNUH) from 2010 to
2014. We reviewed the patients' demographics and oncologic and reconstructive data after receiving approval from the Institutional Review Board of Seoul National University Hospital (IRB No. H-1602-132-744). We excluded patients with pathologic results indicating phyllodes tumor, angiosarcoma, or metastatic cancer at initial presentation; those who underwent prophylactic mastectomy; and those with prior history of breast cancer. We excluded patients with major complications such as flap loss or implant loss that may delay adequate postoperative anti-cancer treatment to achieve pure oncologic comparisons of the two reconstruction methods. In other words, we assumed that all implant or flap reconstructions were properly performed, assessing oncologic effects of each reconstruction method in terms of hemodynamic or immunologic flux, rather than focusing on pragmatic outcomes. Cases converted to flap reconstruction after tissue expander insertion were also excluded. The primary endpoint in our study was locoregional recurrence or distant metastasis.

\section{Comparison of clinical outcomes using propensity-score matching}

We grouped the patients into two cohorts according to IBR method: (1) patients who underwent IBR with implant (including tissue expander), and (2) patients who underwent IBR with flap transfer. For the comparison of oncologic outcomes between the two IBR methods, we conducted propensity score matching. We calculated the propensity scores by logistic regression analysis including age, American Joint Committee on Cancer (AJCC) 7 th ed. tumor staging [20], and estrogen receptor (ER) status. We matched patients by propensity score using the nearest-neighbor method with a matching ratio of 1 : 1. The caliper width was equal to 0.2 times the standard deviation of the logit of the propensity score. After matching, we reviewed the covariate balance for statistical significance and standardized difference.

\section{Immunohistochemistry}

ER, progesterone receptor (PR), human epidermal growth factor receptor-2 (HER2), histologic grade (HG), nuclear grade (NG), and Ki-67 expressions were evaluated. ER, PR and HER2 was evaluated following ASCO/ CAP Guideline [21-23]. HG was graded according to Nottingham classification [24]. The percentage of Ki-67 was determined by the number of $\mathrm{Ki}-67$ positive cells among the total number of counted tumor cells. High expression of Ki-67 was defined as $\geq 10 \%$, based on the previous study in our institution $[25,26]$.

\section{Operative technique}

We performed IBR using implant or flap after mastectomy. In the implant-based reconstructions, we inserted 
an implant (or tissue expander) according to the amount of skin resected during the mastectomy. We inserted the implant at the submuscular layer and used an acellular dermal matrix (ADM) [CG CryoDerm (CGBio Corp., Seongnam, Korea) or DermACELL (LifeNet Health, Virginia Beach, VA, USA)] to cover the inferolateral aspect. In the flap reconstructions, we transferred either a free vascularized flap or a pedicled flap (e.g., from the rectus abdominis or latissimus dorsi myocutaneous flap).

\section{Statistical analysis}

Statistical analyses of $2 \times 2$ contingency tables of categorical variables were performed as appropriate using Fisher's exact test or Pearson's $x^{2}$ test. We calculated the mean durations of survival using the Kaplan-Meier method. Comparisons between groups were performed using log-rank tests. We used logistic regression and a Cox regression model to analyze the effects of continuous numeric variables on clinical outcomes. Multivariate analysis was achieved using logistic regression and Cox regression with factors that showed $p$-values $<0.1$ in the univariate analyses. Locoregional relapse-free interval (LRRFI) was defined as the time between the breast cancer surgery and the detection of locoregional recurrence by biopsy or imaging. Disease-free interval (DFI) was defined as the time between breast cancer surgery and the detection of any relapse. We focused on DFI rather than DFS because there were a few deaths not related to breast cancer which would askew oncologic outcome in this subset. All statistical tests were two-sided with $p<$ 0.05 as the threshold for statistical significance. Analyses were performed using the Statistical Package for the Social Sciences for Windows Version 21.0 (IBM, Chicago, IL, USA).

\section{Results}

\section{Baseline characteristics and propensity score matching} Between January 2010 and December 2014, 731 patients underwent IBR after mastectomy at SNUH for primary breast cancer. A total of 664 patients who meet study criteria underwent propensity score matching based on age, cancer stage [20], and ER status, which resulted in the inclusion of 496 patients (247 implants and 249 flaps) for further analysis. (Additional file 1: Fig. S1).

There were no differences between the two groups in cancer stage, weight of excised breast mass, chemotherapy or radiotherapy status, axillary lymph node status, ER status, PR status, NG, HG, and HER2 amplification after propensity score matching (Table 1 , Additional file 2: Table S1). Of the 247 patients from implant group, 60 (24.3\%) patients received implant insertion, and 187 (75.7\%) received tissue expander insertion. The majority of flap reconstructions used a free transverse rectus abdominis myocutaneous (TRAM) flap $(n=238,95.6 \%)$; other used a pedicled latissimus dorsi myocoutaneous flap $(n=7,2.8 \%)$, a free superficial inferior epigastric artery perforator flap $(n=1,0.4 \%)$, a free inferior gluteal artery perforator flap $(\mathrm{n}=1,0.4 \%)$, a free gracilis flap $(n=1,0.4 \%)$, or a free lumbar artery perforator flap $(\mathrm{n}=$ $1,0.4 \%)$.

\section{Cancer recurrence after reconstruction}

During median follow-up duration was 58.2 months (57.3 and 58.3 months for implant and flap group, respectively) there were 38 recurrence events. Cancer stage was an independent prognostic factor for recurrence $(p<0.001)$. The NG $(p=0.004)$, HG $(p=0.001)$, and Ki$67(p<0.001)$ were also prognostic factors for cancer recurrence. Vascular emboli and lymphatic emboli affected the DFI $(p<0.001$ and $p<0.001$, respectively); however, ER status $(p=0.172)$, PR status $(p=0.190)$, and HER-2 status $(p=0.642)$ did not.

There was no difference in the DFI between implant group and flap group. During follow up, 14 patients relapsed in implant group and 24 patients relapsed in flap group. The 5-year DFI rate was $93 \%$ in implant group and $90 \%$ in flap group $(p=0.100)$ (Fig. 1a). There were no differences in DFI between the patients that underwent one-stage implant and two-stage expander insertion $(p=0.861)$ or between those that underwent TRAM flap and other types of flap reconstructions $(p=0.859)$.

In a multivariate analysis for DFI including cancer stage, NG, HG, and Ki-67, cancer stage $(p=0.007)$ was an independent prognostic factor (Additional file 3: Table S2).

\section{Systemic cancer recurrence affected by IBR method in aggressive tumors}

When we considered the different cancer stages separately, there was no difference in DFI between the implant and flap group ( $p$-value for stage $1=0.642$; stage $2=0.195$; stage $3=0.132$ ) (Fig. 2).

On the other hand, when we considered the HG separately, patients with HG 3 (high HG) in flap group $(n=74)$ had a lower 5 -year DFI rate than implant group $(n=75)$ (5-year DFI rate for implant group 92\% vs. flap group $77 \% ; p=0.012$ ). There was no such difference among the patients with HG 1 or 2 , however $(p=0.917)$. Likewise, flap reconstruction showed short DFI in patients with high Ki-67 $(p=0.028)$. In contrast, there was no difference in DFI between the two groups in low Ki-67 $(p=0.278)$. When both HG and Ki-67 were considered, aggressive tumor (defined by high HG and high Ki-67) relapsed more frequently following flap reconstruction than implant reconstruction ( $p=0.004)$ (Fig. 3a-d). Patient characteristics between the two reconstruction groups in high HG and/ or high Ki-67 group did not differ. 
Table 1 Patients demographics

\begin{tabular}{llll}
\hline Characteristics & & Reconstruction type & \\
\cline { 4 - 4 } & & Implant* $(n=247)$ & \\
\hline Age, years & Average & $41 \pm 8.73$ & $43 \pm$ \\
Diagnosis & & & 172 \\
& IDC & 164 & 50 \\
DCIS & 46 & 8 \\
ILD & 17 & 4 \\
LCIS & 0 & 8 \\
& Mixed & 11 & 7 \\
& Others & 9 &
\end{tabular}

Mastectomy type

$\begin{array}{ll}\text { TM } & 115 \\ \text { SSM } & 64 \\ \text { NSM } & 68\end{array}$

Excised breast mass, gram

Lymph node status

AJCC Stage

$\begin{array}{lll}0 & 47 & 57 \\ \text { IA } & 99 & 82 \\ \text { IB } & 1 & 0 \\ \text { IIA } & 49 & 53 \\ \text { IIB } & 24 & 26 \\ \text { IIIA } & 20 & 19 \\ \text { III } & 0 & 3 \\ \text { IIC } & 7 & 9\end{array}$

Chemotherapy

none 111

Radiotherapy

none
adjuvant

Nuclear grade, no. (\%)

Histological grade, no. (\%)

16
104
74


Table 1 Patients demographics (Continued)

\begin{tabular}{|c|c|c|c|c|}
\hline \multirow[t]{2}{*}{ Characteristics } & & \multicolumn{2}{|l|}{ Reconstruction type } & \multirow[t]{2}{*}{$p$-value } \\
\hline & & Implant* $(n=247)$ & Flap $(n=249)$ & \\
\hline \multirow[t]{3}{*}{ ER, no. (\%) } & & & & 0.462 \\
\hline & Negative & 49 & 43 & \\
\hline & Positive & 198 & 206 & \\
\hline \multirow[t]{3}{*}{ PR, no. (\%) } & & & & 0.952 \\
\hline & Negative & 76 & 76 & \\
\hline & Positive & 171 & 173 & \\
\hline \multirow[t]{3}{*}{ HER2, no. (\%) } & & & & 0.128 \\
\hline & Negative & 174 & 193 & \\
\hline & Positive & 56 & 44 & \\
\hline \multirow[t]{3}{*}{ Ki67, no. (\%) } & & & & 0.014 \\
\hline & $<10 \%$ & 198 & 174 & \\
\hline & $\geq 10 \%$ & 48 & 71 & \\
\hline
\end{tabular}

IDC invasive ductal carcinoma; ILC invasive lobular carcinoma; DCIS ductal carcinoma in situ; LCIS lobular carcinoma in situ; TM total mastectomy; SSM skin-sparing mastectomy; NSM nipple-sparing mastectomy; AJCC American Joint Committee on Cancer; ER estrogen receptor; PR progesterone receptor; HER2 human epidermal growth factor receptor-2

*Implant group includes patients who received reconstruction with tissue expander

In multivariate analysis for DFI performed within high HG group considering cancer stage, hormone receptor (HR), HER2 and reconstruction type, the reconstruction type was the independent prognostic factor $(p=0.018)$ (Table 2). Likewise, in high Ki-67 group, the reconstruction type was the independent prognostic factor for DFI in multivariate analysis ( $p=0.015$, data not shown).

When HR and HER2 status was considered, DFI was not different between two groups in each tumor subtype: including triple-negative breast cancer (TNBC; $p=$ $0.668)$, and HR-positive breast cancer $(p=0.230)$. However, in 71 aggressive tumors (high HG and high Ki-67), frequent relapse after flap reconstruction was seen especially in HR-positive breast cancers (HR-positive: $p=$ 0.008; HR-positive/HER2-negative: $p=0.002$ ), which accounts for majority of our study population (Fig. 3e-f).
Next, we observed whether the reconstruction type affected locoregional recurrence. There were 20 locoregional recurrences during follow up: 9 in implant and 11 in flap group. The 5-year LRRFI rate was $95 \%$ in implant group and $95 \%$ in flap group $(p=0.991)$ (Fig. 1b). Unlike the DFI, the LRRFI was not affected by reconstruction method neither in high HG tumor $(p=0.445)$ nor in high Ki-67 tumor $(p=0.791)$. The reconstruction type did not affect locoregional recurrence in a multivariate analysis $(p=0.704)$.

\section{Discussion}

IBR is widely performed and previous studies have tried to establish the oncologic safety of IBR, showing that IBR did not increase recurrence rates or delay the detection of recurrence $[3,4]$. However, those previous
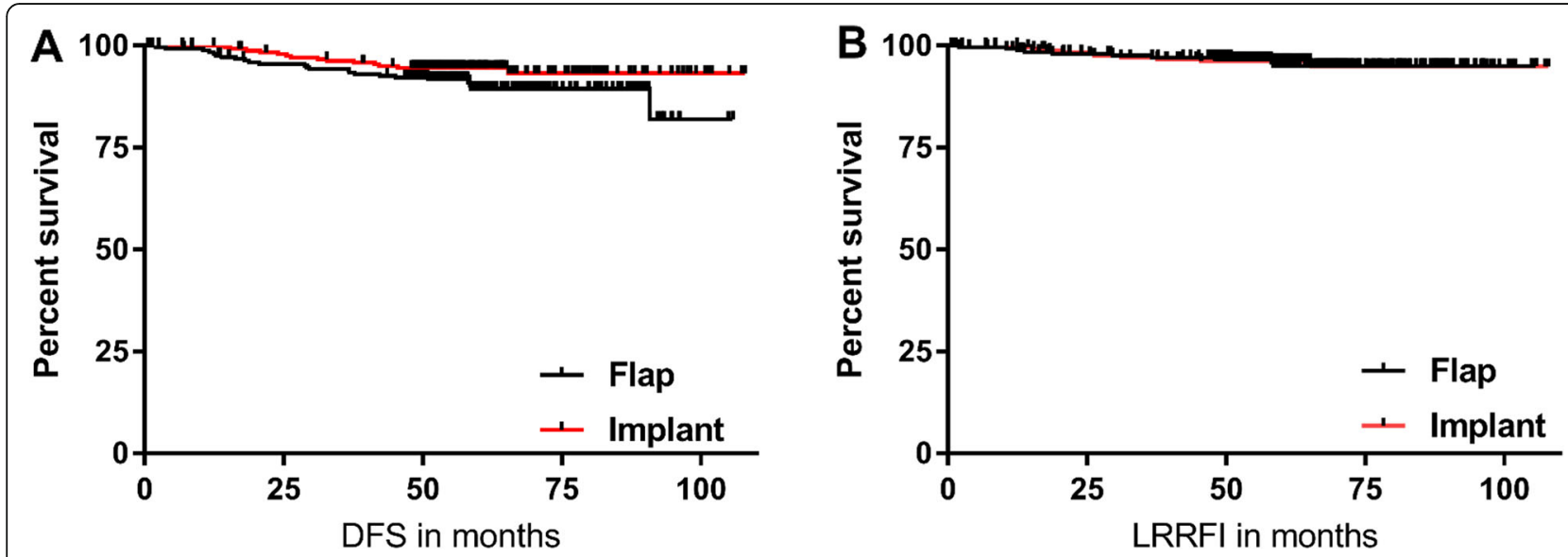

Fig. 1 Disease free survival (a) and Locoregional relapse free interval (b) in implant and flap based immediate breast reconstruction 

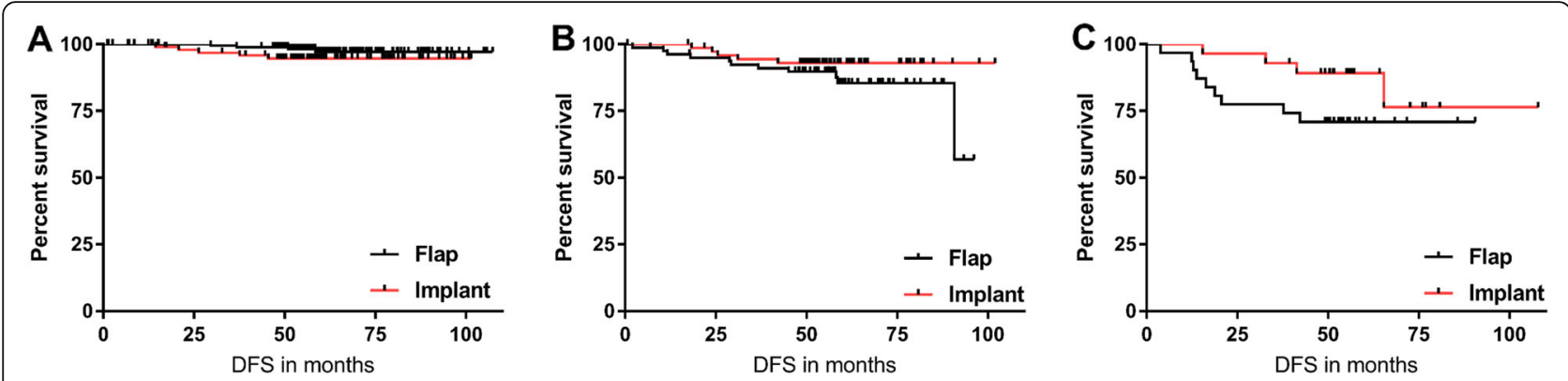

Fig. 2 Disease free survival in (a) Stage I, (b) Stage II, and (c) Stage III patients according to breast reconstruction type

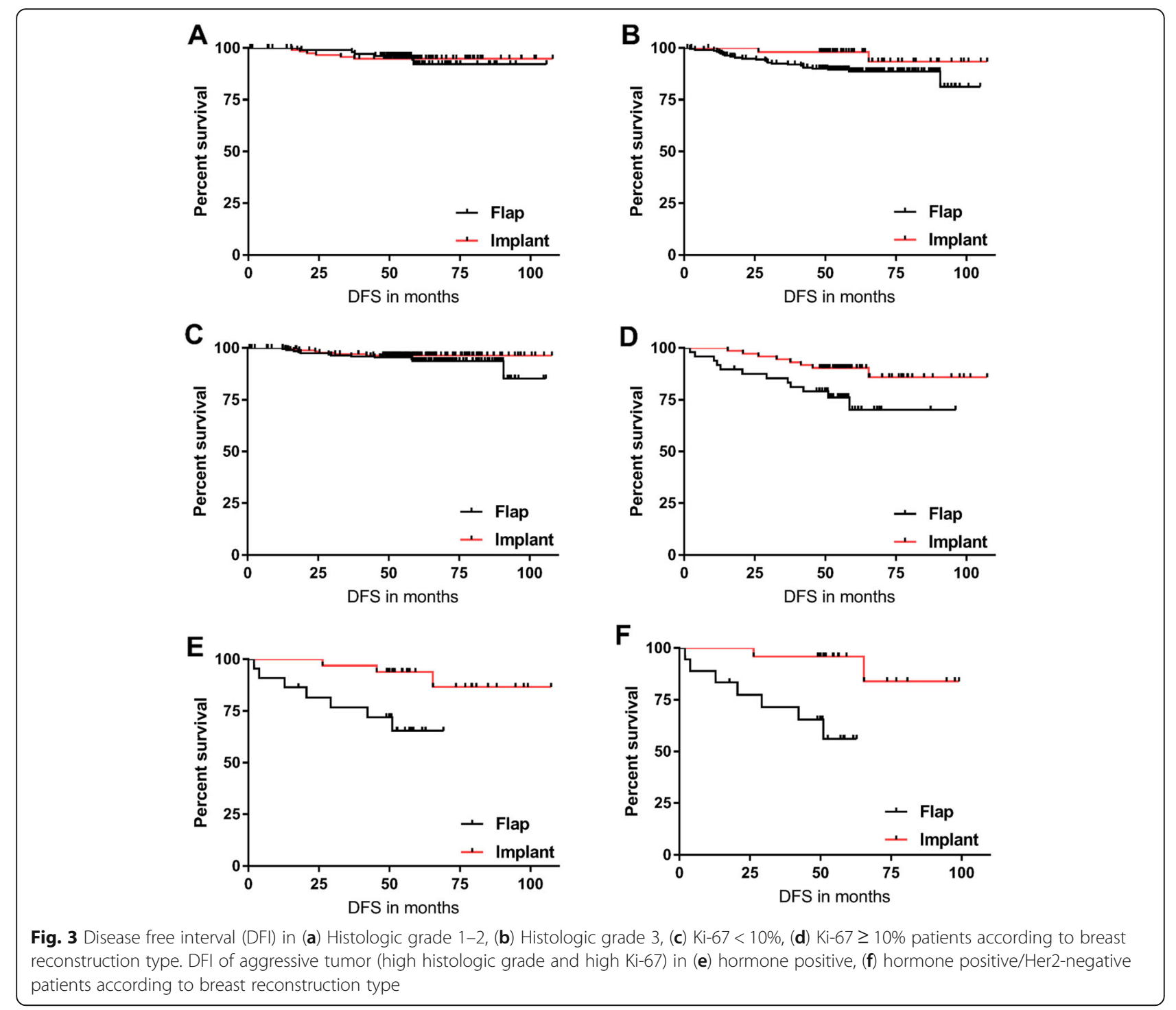


Table 2 Hazard ratio and p-value of disease-free interval in high histologic grade (grade 3) group using a Cox proportional hazard model in multivariate analysis

\begin{tabular}{|c|c|c|c|c|}
\hline \multirow[t]{2}{*}{ Characteristics } & \multirow[t]{2}{*}{$\mathrm{HR}$} & \multicolumn{3}{|l|}{$95 \% \mathrm{Cl}$} \\
\hline & & Lower & Upper & $p$-value \\
\hline Reconstruction type & & & & 0.018 \\
\hline Prosthesis & 1.00 & & & \\
\hline Flap & 3.39 & 1.23 & 9.32 & \\
\hline Stage & & & & 0.040 \\
\hline 1 & 1.00 & & & \\
\hline$\|$ & 1.79 & 0.56 & 5.78 & \\
\hline III & 4.55 & 1.32 & 15.67 & \\
\hline Hormone receptor & & & & 0.491 \\
\hline Positive & 1.00 & & & \\
\hline Negative & 1.42 & 0.52 & 9.32 & \\
\hline HER2 & & & & 0.430 \\
\hline Positive & 1.52 & 0.54 & 4.33 & \\
\hline Negative & 1.00 & & & \\
\hline
\end{tabular}

HR Hazard ratio; $\mathrm{Cl}$ confidence interval; HER2 human epidermal growth factor receptor-2

reports did not differentiate the outcomes according to specific IBR methods or tumor histologies $[3,5,6]$. To our knowledge, no one has yet performed a matched case-control study comparing locoregional and distant metastasis rates between reconstruction methods. There may be, however, a chance of tumor spreading through vascular anastomosis of flap with surrounding breast envelope, raising doubts about oncologic safety. The hemodynamic environment, which may influence tumor spreading, is presumed to be different between the two reconstruction methods. Hence, although neither method is reported to increase the relapse risk in general, the oncologic safety of flap reconstruction still needs to be more precisely analyzed from diverse perspectives.

In this regard, we performed a propensity-matched, case-control study to compare oncologic safety between implant-based and flap reconstructions: we focused on DFI rather than DFS because there were a few deaths not related to breast cancer which would askew oncologic outcome in this subset. We excluded patients with major complications that may delay adequate postoperative anti-cancer treatment to achieve pure oncologic comparisons of the two reconstruction methods. In other words, we assumed that all implant or flap reconstructions were properly performed, assessing oncologic effects of each reconstruction method in terms of hemodynamic or immunologic flux, rather than focusing on pragmatic outcomes. We did not find any difference in DFI between the two reconstruction methods. We can therefore conclude that in general, the choice of reconstruction method does not affect oncological outcome. In addition, there were some unexpected findings in our subgroup analyses.

Among patients with high HG, the DFI was shorter in the flap group than in the implant group. In line with histologic grading, patients with high Ki-67 showed shorter tendency for DFI in flap group compared to implant group. This was confirmed both in univariate and multivariate analysis. When we combined HG and Ki-67 to define tumor aggressiveness, aggressive tumor showed higher rate of relapse following flap reconstruction than implant-based reconstruction. On the other hand, locoregional recurrence was not different based on reconstruction method among patients with aggressive histology suggesting that flap reconstruction was related to the systemic recurrence. There was no difference in chemotherapy status or cancer stage between the two reconstruction groups with high HG tumor $(n=148)$, which excludes the possibility of selection bias in that finding. Due to small number of absolute relapse events, affirmative conclusion could not be drawn with this single study. However, it should be noted from this study that high HG tumor may have increased rate of systemic relapse with flap reconstruction which accompanies increased vascularity around surgical bed. Surgical stress imposed by flap operations may thus foster distant relapse of aggressive tumors, as in a mouse model of breast cancer [27]. Because perioperative immunomodulation, stemming from surgical stress, may figure prominently in antimetastatic immune activity $[28,29]$, it is not surprising that flap reconstruction, which entails prolonged operative time and therefore more surgical stress than implant reconstruction, is associated with shorter DFIs. Distant recurrences may be cultivated from preexisting micrometastases in aggressive cancer cell types due to perioperative immunosuppression [28, 29]. In fact, HG [30] and Ki-67 [31] are well-known prognostic factors for breast cancer. But we could not find similar findings as ours in the literature and concluded that it was because previous research did not focus on HG or Ki-67. Eriksen et al. [6], Howard et al. [3], and McCarthy et al. [5] all analyzed the oncologic outcomes of implant or flap-based IBR. However, data regarding the HG and Ki-67 were not assessed in all studies. It should be noted that, on the other hand, DFI was not different depending on the reconstruction method when the analysis was stratified on other tumor characteristics such as cancer stage, nuclear emboli, or TNBC. This suggests that the tumor aggressiveness at the single-cell level represented by high HG or high $\mathrm{Ki}-67$ is the most important factor in the increased risk of relapse after flap reconstruction in breast cancer.

We tried to overcome limitations of our study design. First, there was a possibility of bias in the patient characteristics between the two groups. Young patients may more likely want to undergo implant-based reconstruction for simultaneous augmentation of contralateral breast. On 
the other hand, surgeons might prefer flap reconstruction in advanced cancer, considering the possible radiationrelated complications associated with implants. We tried to minimize this bias by using propensity score matching for more balanced comparison. Second, majority of our study population were HR-positive and HER2-negative. We could observe short DFI after flap reconstruction in aggressive tumor cell phenotype within this subgroup but not in other subgroups with statistical significance. Hence, effect on recurrence according to reconstruction method in less representative subtypes in our study should be analyzed in further studies. Lastly, because this was a retrospective study, we cannot be certain that some information that might have influenced the outcome was not left out. One example is tumor size, but data concerning tumor size or the ratio of tumor size to breast size were not available for analyses. In addition, how the reconstruction method was chosen is another important factor that could not be assessed because of the retrospective nature of the study. However, as several previous important retrospective studies $[32,33]$, we assume discovering novel findings from retrospective cohort study is virtually not impossible. We hope further study with robust research design (e.g. prospective study) would consolidate our anecdotal findings.

\section{Conclusions}

We report that there is no difference in cancer recurrence according to the method used for immediate breast reconstruction in general. There is, however, a possibility that flap reconstruction increases the risk of systemic recurrence in high HG and/or high Ki-67 tumors. Our study suggests that when breast cancers is revealed to have high HG or high $\mathrm{Ki}-67$ upon preoperative biopsy, flap reconstruction after mastectomy should be performed with caution from an oncologic point of view.

\section{Supplementary information}

Supplementary information accompanies this paper at https://doi.org/10. 1186/s12885-020-6568-2

\section{Additional file 1. Fig. S1. CONSORT flow diagram \\ Additional file 2: Table S1. Patient demographics before propensity score matching}

Additional file3: Table S2. Hazard ratio and $p$-value of disease-free interval using a Cox proportional hazard model in multivariate analysis

\footnotetext{
Abbreviations

AJCC: American Joint Committee on Cancer, 7th ed; DFS: Disease-free survival; ER: Estrogen receptor; HER2: Human epidermal growth factor receptor 2; HG: Histologic grade; HR: Hormone receptor; IBR: Immediate breast reconstruction; LRRFI: Locoregional recurrence-free interval; NAC: Nipple-areola complex; NG: Nuclear grade; NSM: Nipple-sparing mastectomy; PR: Progesterone receptor; SSM: Skin-sparing mastectomy; TM: Total mastectomy; TRAM: Transverse rectus abdominis myocutaneous
}

\section{Acknowledgements}

The authors would like to express sincere gratitude to Youngil Koh for providing oncologic insight and Do Young Yoon for helping with propensity score matching.

\section{Authors' contributions}

JHH collected clinical data, analyzed and interpreted the data, wrote the main manuscript text. KYH collected clinical data, analyzed the data and wrote the main manuscript text. HL contributed to the oncologic analysis and interpretation of the data. HM, WH, DN collected the patients' clinicopathological records and played critical roles in oncologic interpretation of the data. JL, SY, and HC collected the patients' information and prepared the figures and participated in analysis. USJ supervised the manuscript and study design, and played critical roles in interpretation of data. All authors reviewed the manuscript and are accountable for the work.

\section{Funding}

N/A

\section{Availability of data and materials}

All data generated or analyzed during this study are included in this published article and its supplementary information files.

\section{Ethics approval and consent to participate}

The study was approved by the Institutional Review Board of Seoul National University Hospital (IRB No. H-1602-132-744). Waivers of informed consent was approved by the Institutional Review board.

\section{Consent for publication}

N/A

\section{Competing interests}

The authors declare that they have no competing interests.

\section{Author details}

${ }^{1}$ Department of Plastic and Reconstructive Surgery, Seoul National University Hospital, Seoul, South Korea. ${ }^{2}$ Department of Plastic and Reconstructive Surgery, Dongguk University Medical Center, Goyang, South Korea. ${ }^{3}$ Department of Surgery, Seoul National University College of Medicine, Seoul, South Korea. ${ }^{4}$ Cancer Research Institute, Seoul National University, Seoul, South Korea. ${ }^{5}$ Department of Plastic and Reconstructive Surgery, Seoul National University College of Medicine, Seoul, South Korea.

Received: 24 May 2019 Accepted: 21 January 2020

Published online: 30 January 2020

\section{References}

1. Panchal H, Matros E. Current trends in Postmastectomy breast reconstruction. Plast Reconstr Surg. 2017;140:75-13S.

2. Xavier Harmeling J, et al. The effect of immediate breast reconstruction on the timing of adjuvant chemotherapy: a systematic review. Breast Cancer Res Treat. 2015;153:241-51.

3. Howard MA, et al. Breast cancer local recurrence after mastectomy and TRAM flap reconstruction: incidence and treatment options. Plast Reconstr Surg. 2006;117:1381-6.

4. Patani N, Devalia H, Anderson A, Mokbel K. Oncological safety and patient satisfaction with skin-sparing mastectomy and immediate breast reconstruction. Surg Oncol. 2008:17:97-105.

5. McCarthy $\mathrm{CM}$, et al. Breast cancer recurrence following prosthetic, postmastectomy reconstruction: incidence, detection, and treatment. Plast Reconstr Surg. 2008;121:381-8.

6. Eriksen C, et al. Immediate reconstruction with implants in women with invasive breast cancer does not affect oncological safety in a matched cohort study. Breast Cancer Res Treat. 2011;127:439-46.

7. Elder EE, et al. Quality of life and patient satisfaction in breast cancer patients after immediate breast reconstruction: a prospective study. Breast. 2005;14:201-8.

8. Berbers J, et al. Reconstruction: before or after postmastectomy radiotherapy? A systematic review of the literature. Eur J Cancer. 2014:50: 2752-62. 
9. Godfrey PM, Godfrey NV, Romita MC. Immediate autogenous breast reconstruction in clinically advanced disease. Plast Reconstr Surg. 1995;95: 1039-44.

10. Zhang $P$, et al. Comparison of immediate breast reconstruction after mastectomy and mastectomy alone for breast cancer: a meta-analysis. Eur J Surg Oncol. 2017:43:285-93.

11. Gieni $M$, et al. Local breast cancer recurrence after mastectomy and immediate breast reconstruction for invasive cancer: a meta-analysis. Breast. 2012;21:230-6.

12. Hidalgo DA, et al. Immediate reconstruction after complete skin-sparing mastectomy with autologous tissue. J Am Coll Surg. 1998;187:17-21.

13. Disa JJ, et al. Skin-sparing mastectomy and immediate autologous tissue reconstruction after whole-breast irradiation. Plast Reconstr Surg. 2003;111: $118-24$.

14. Nelson JA, Disa JJ. Breast reconstruction and radiation therapy: an update. Plast Reconstr Surg. 2017;140:60S-8S.

15. El-Sabawi B, Sosin M, Carey JN, Nahabedian MY, Patel KM. Breast reconstruction and adjuvant therapy: a systematic review of surgical outcomes. J Surg Oncol. 2015;112:458-64.

16. Newman LA, et al. Feasibility of immediate breast reconstruction for locally advanced breast cancer. Ann Surg Oncol. 1999;6:671-5.

17. Carlson GW. Breast reconstruction. Surgical options and patient selection. Cancer. 1994;74:436-9.

18. Park SH, et al. Oncologic safety of immediate breast reconstruction for invasive breast Cancer patients: a matched case control study. J Breast Cancer. 2016;19:68-75

19. Isern $A E$, et al. Risk of recurrence following delayed large flap reconstruction after mastectomy for breast cancer. Br J Surg. 2011;98:659-66.

20. Edge SB. American Joint Committee on Cancer. \& American Cancer Society. In: AJCC cancer staging handbook : from the AJCC cancer staging manual. New York: Springer; 2010.

21. Wolff AC, et al. Recommendations for human epidermal growth factor receptor 2 testing in breast cancer: American Society of Clinical Oncology/ College of American Pathologists clinical practice guideline update. Archives pathology laboratory medicine. 2014;138:241-56.

22. Hammond ME, et al. American Society of Clinical Oncology/college of American pathologists guideline recommendations for immunohistochemical testing of estrogen and progesterone receptors in breast cancer. J Clin Oncol. 2010;28:2784-95.

23. Perez EA, et al. HER2 testing in patients with breast cancer: poor correlation between weak positivity by immunohistochemistry and gene amplification by fluorescence in situ hybridization. Mayo Clin Proc. 2002;77:148-54

24. Elston CW, Ellis IO. Pathological prognostic factors in breast cancer. I The value of histological grade in breast cancer: experience from a large study with long-term follow-up. Histopathology. 1991;19:403-10.

25. Jung SY, et al. Ki-67 expression gives additional prognostic information on St. Gallen 2007 and adjuvant! Online risk categories in early breast cancer. Ann Surg Oncol. 2009;16:1112-21.

26. Keam B, et al. Ki-67 can be used for further classification of triple negative breast cancer into two subtypes with different response and prognosis. Breast Cancer Res. 2011;13:R22.

27. Le CP, et al. Chronic stress in mice remodels lymph vasculature to promote tumour cell dissemination. Nat Commun. 2016;7:10634.

28. Neeman E, Zmora O, Ben-Eliyahu S. A new approach to reducing postsurgical cancer recurrence: perioperative targeting of catecholamines and prostaglandins. Clin Cancer Res. 2012;18:4895-902.

29. Zhou $L$, et al. Propranolol attenuates surgical stress-induced elevation of the regulatory $T$ cell response in patients undergoing radical mastectomy. J Immunol. 2016;196:3460-9.

30. Henson DE, Ries L, Freedman LS, Carriaga M. Relationship among outcome, stage of disease, and histologic grade for 22,616 cases of breast cancer. The basis for a prognostic index. Cancer. 1991;68:2142-9.

31. Duffy MJ, et al. Clinical use of biomarkers in breast cancer: updated guidelines from the European group on tumor markers (EGTM). Eur J Cancer. 2017;75:284-98.

32. Hong KY, et al. Opportunistic biopsy of internal mammary lymph nodes during immediate breast reconstruction after mastectomy for breast malignancies. Ann Surg Oncol. 2017;24:1881-8.
33. Lhenaff $M$, et al. A single-center study on total mastectomy versus skinsparing mastectomy in case of pure ductal carcinoma in situ of the breast. Eur J Surg Oncol. 2019;45:950-955.

\section{Publisher's Note}

Springer Nature remains neutral with regard to jurisdictional claims in published maps and institutional affiliations.
Ready to submit your research? Choose BMC and benefit from:

- fast, convenient online submission

- thorough peer review by experienced researchers in your field

- rapid publication on acceptance

- support for research data, including large and complex data types

- gold Open Access which fosters wider collaboration and increased citations

- maximum visibility for your research: over $100 \mathrm{M}$ website views per year

At BMC, research is always in progress.

Learn more biomedcentral.com/submissions 